\title{
An Efficient Synthesis of Thiazolo and Thiadiazolo Quinoxaline Derivatives in Ionic Liquid
}

\author{
B. PRASANNA*, B. SRINIVAS, Y. JAGANNADHAM and SUMANGALA RAO
}

Research Centre, Department of Chemistry

Chaitanya PG College, Warangal-506 001, India

prasschem@gmail.com

Received 21 July 2011; Accepted 5 September 2011

\begin{abstract}
A series of 3-substitutedphenyl-1-thia-tetrazopentaleno[1,2-b] naphthalene 4(a-d) and 2-substitutedphenyl-1-thia-pentazopentaleno[1,2-b] naphthalene 5(a-d) were synthesized via., the reaction of 2-aminothiazoles 2(a-d) and 2-aminothiadiazoles 3(a-d) with 2,3-dichloro quinoxaline $\mathbf{1}$ in ionic liquid without using any catalyst. This protocol has the advantages of easier workup, milder reaction conditions, high yields, and environmentally benign procedure over traditional methods. The synthesized compounds 4(a-d) and 5(a-d) tested for their anti-fungal activity and these compounds were characterized by IR, NMR and Mass spectral analysis.
\end{abstract}

Keywords: Aminothiazoles, Aminothiadiazoles, Dichloroquinoxaline.

\section{Introduction}

Compounds containing the quinoxaline nucleus exhibit abroad spectrum of biological activity such as, anti-viral ${ }^{1}$, antiinflammatory, antiprotozoal ${ }^{2}$, antihelminthic ${ }^{3}$, anticancer ${ }^{4}$, antimalarial $^{5}$, and antidepressant activities ${ }^{6}$. Some of the antibiotics such as levomycin, actinoleutin, and echinomycin also contain a quinoxaline scaffold and these are known to inhibit the growth of gram positive bacteria ${ }^{7}$ and are active against various transplantable tumours $^{8}$. Besides the biological activities, quinoxalines are also used in the synthesis of organic semiconductors, dyes ${ }^{9}$, and electroluminescent materials ${ }^{10}$. Some of quinoxalines exhibit potent central nervous system (CNS) activities such as analgesic and antiinflammatory activities ${ }^{11}$. Few of azolopyrimidoquinaxalines, pyrimidoquinazolines exhibited good antioxidants, anti-inflammatory, and analgesic activities ${ }^{12-15}$. Furthermore, organic compounds bearing thiazolopyrimidines and pyridothiazolo quinoxaline nuclei were found to possess potent anticancer activities ${ }^{16}$. These biological applications and development of new route to heterocyclic systems in ionic liquid prompted us to synthesize some new heterocyclic derivatives having phenylthiazoles, thiadiazoles, and quinoxaline moieties starting from 2, 3-dichloro quinoxaline in ionic liquid without using any catalyst and search of better anti-fungal activity. (Schemes $1 \& 2$ ). 


\section{Experimental}

Melting points were measured in open capillary on Buchi melting point B-540 apparatus and were uncorrected. IR spectra were recorded on Simadzu FTIR-8400 spectrometer using KBr pellets. ${ }^{1} \mathrm{H}$ NMR $(300 \mathrm{MHz})$ spectra recorded in DMSO- $d_{6}$ on a Bruker AVANCE 300 instrument with the TMS as an internal standard. All the chemical shifts values were recorded as $\delta \mathrm{ppm}$. Mass spectra (EI-MS) were taken on Perkin Elmer (SCIEX API-2000, ESI) at $12.5 \mathrm{eV}$. CHNS analysis was carried out on Carlo Erba E A 1108 automatic analyzer. The progress of each reaction was monitored and purity of the compounds was checked by thin layer chromatography.

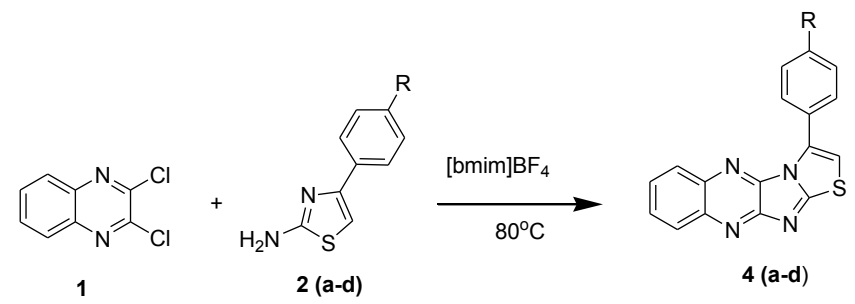

Scheme 1

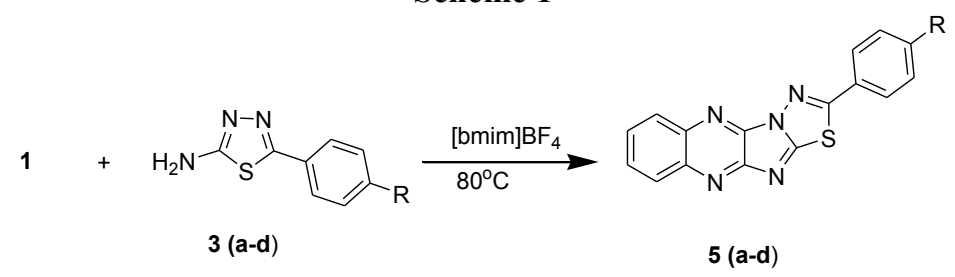

$\mathrm{R}=\mathrm{H}, \mathrm{Cl}, \mathrm{Br}, \mathrm{CH}_{3}$

Scheme 2

General Procedure for the Synthesis of 3-Substitutedphenyl-1-thiatetrazopentaleno-[1,2-b]naphthalene $4(a-d)$ and 2-substituted phenyl-1-thiapentazopentaleno[1,2-b] naphthalene 5(a-d)

A dry $50 \mathrm{~mL}$ flask was charged with 2, 3-dichloroquinoxaline ${ }^{17-18} \mathbf{1}(1 \mathrm{mmol})$ and substituted 2-aminothiazoles 2(a-d) (1 mmol)/2-aminothiadiazoles 3(a-d) $(1 \mathrm{mmol})$ and ionic liquid [bmim] $\mathrm{BF}_{4}(2 \mathrm{~mL})$. Then the reaction mixture was stirred for $1.5 \mathrm{~h}$ to $2.5 \mathrm{~h}$ to complete the reaction (monitored by TLC) at $80^{\circ} \mathrm{C}$. The reaction mixture was cooled to room temperature and poured into $10 \mathrm{~mL}$ of water. The solid product collected by filtration and re-crystallized from methanol to give pure compounds $\mathbf{4}(\mathbf{a}-\mathbf{d})$ and $\mathbf{5 ( a - d ) .}$ The filtration was washed with acetic ester for several times concentrated under reduced pressure at $100^{\circ} \mathrm{C}$ for several hours to give reusable solvent. A similar procedure was used in preparing all the compounds.

\section{Spectral Data of the Synthesized Compounds 4(a-d) and 5(a-d)}

3-Phenyl-1-thia-3a,4,9,10-tetrazopentaleno[1,2-b]naphthalene (4a)

Mp: $240-242^{0} \mathrm{C}$; IR (KBr): v $\left(\mathrm{cm}^{-1}\right) 1612(\mathrm{C}=\mathrm{N}) ;{ }^{1} \mathrm{H}$ NMR (300 MHz, DMSO-d $\left.d_{6}\right): \delta 7.12$ (s, $1 \mathrm{H}, \mathrm{CH}), 7.22-7.40(\mathrm{~m}, 5 \mathrm{H}, \mathrm{Ar}-\mathrm{H})$ 7.48-7.52 (dd, $1 \mathrm{H}$, quin-H), $7.80(\mathrm{dd}, 1 \mathrm{H}$, quin-H), 8.12 (dd, $2 \mathrm{H}$, quin-H). EI-MS: $303[\mathrm{M}+1]^{+}$. Anal.Calcd for $\mathrm{C}_{17} \mathrm{H}_{10} \mathrm{~N}_{4} \mathrm{~S}: \mathrm{C}, 67.53 ; \mathrm{H}, 3.33 ; \mathrm{N}$, $18.53 \%$. Found: C, 67.56; H, 3.31; N, 18.51\%. 


\section{3-(4-Chlorophenyl)-1-thia-3a,4,9,10-tetrazopentaleno[1,2-b]naphthalene(4b)}

Mp: $232-233^{0} \mathrm{C}$; IR (KBr): v $\left(\mathrm{cm}^{-1}\right) 1618(\mathrm{C}=\mathrm{N}) ;{ }^{1} \mathrm{H}$ NMR $\left(300 \mathrm{MHz}\right.$; DMSO- $\left.d_{6}\right): \delta$ 7.027.18 (m, 4H, Ar-H), 7.20 (s, 1H, CH), 7.42 (dd, 1H, quin-H), 7.80( dd, 1H, quin-H), 8.128.14 (dd, 2H, quin-H). EI-MS: $338[\mathrm{M}+1]^{+}$. Anal. Calcd for $\mathrm{C}_{17} \mathrm{H}_{9} \mathrm{ClN}_{4} \mathrm{~S}: \mathrm{C}, 60.62 ; \mathrm{H}, 2.69$, $\mathrm{N}, 16.64 \%$. Found: C, 60.64, H, 2.67, N, 16.62\%.

\section{3-(4-Bromophenyl)-1-thia-3a,4,9,10-tetrazopentaleno[1,2-b]naphthalene(4c)}

Mp: $238-240^{0} \mathrm{C}$; IR(KBr): v $\left(\mathrm{cm}^{-1}\right) 1610(\mathrm{C}=\mathrm{N})$; ${ }^{1} \mathrm{H}$ NMR $\left(300 \mathrm{MHz}, \mathrm{DMSO}-d_{6}\right): \delta 7.16-$ 7.34 (m, 4H, Ar-H), 7.20 (s, 1H, CH), 7.54 (dd, 1H, quin-H), 7.86 ( dd, 1H, quin-H), 8.14$8.18\left(\mathrm{dd}, 2 \mathrm{H}\right.$, quin-H). EI-MS: $382[\mathrm{M}+1]^{+}$. Anal. Calcd for $\mathrm{C}_{17} \mathrm{H}_{9} \mathrm{BrN}_{4} \mathrm{~S}: \mathrm{C}, 53.56 ; \mathrm{H}, 2.38$; $\mathrm{N}, 14.70 \%$. Found: C, 53.54; H, 2.40; N, $14.72 \%$.

\section{3-p-Tolyl-1-thia-3a,4,9, 10-tetrazopentaleno[1,2-b]naphthalene (4d)}

Mp: $245-247^{0} \mathrm{C}$; IR (KBr): v $\left(\mathrm{cm}^{-1}\right) 1610(\mathrm{C}=\mathrm{N}) ;{ }^{1} \mathrm{H}$ NMR (300 MHz, DMSO- $\left.d_{6}\right): \delta 2.32$ (s, $\left.3 \mathrm{H}, \mathrm{CH}_{3}\right), 7.04-7.18$ (dd, 2H, Ar-H), 7.24-7.26 (dd, 2H, Ar-H), 7.14 (s, 1H, CH), 7.60-7.64( $\mathrm{dd}, 2 \mathrm{H}$, quin-H), 8.17-8.19 (dd, 2H, quin-H). EI-MS: $317[\mathrm{M}+1]^{+}$. Anal. Calcd for $\mathrm{C}_{18} \mathrm{H}_{12} \mathrm{~N}_{4} \mathrm{~S}$ : C, 68.33; H, 3.82; N, 17.71\%. Found: C, 68.31; H, 3.80; N, 17.69\%.

\section{2-Phenyl-1-thia-3,3a,4,9,10-pentazopentaleno[1,2-b]naphthalene (5a)}

Mp: $246-248^{0} \mathrm{C}$; IR (KBr): v $\left(\mathrm{cm}^{-1}\right) 1612(\mathrm{C}=\mathrm{N})$; ${ }^{1} \mathrm{H}$ NMR $\left(300 \mathrm{MHz}, \mathrm{DMSO}-d_{6}\right): \delta 7.34-$ 7.64 (m, 5H, Ar-H), 7.72-7.74 (dd, 2H, quin-H), 8.14-8.16 (dd, 2H, quin-H). EI-MS: 304 $[\mathrm{M}+1]^{+}$. Anal. Calcd for $\mathrm{C}_{16} \mathrm{H}_{9} \mathrm{~N}_{5} \mathrm{~S}$ : C, 63.32; H, 2.99; N, 23.09\%. Found: C, 63.32; H, 2.97; $\mathrm{N}, 23.11 \%$.

\section{2-(4-Chlorophenyl)-1-thia-3,3a,4,9,10-pentazopentaleno[1,2-b]naphthalene (5b)}

Mp: $250-251^{0} \mathrm{C}$; IR (KBr): v $\left(\mathrm{cm}^{-1}\right) 1618(\mathrm{C}=\mathrm{N}) ;{ }^{1} \mathrm{H}$ NMR $\left(300 \mathrm{MHz}, \mathrm{DMSO}-d_{6}\right): \delta 7.38-$ 7.42 (dd, 2H, Ar-H), 7.74-7.76 (dd, 2H, Ar-H), 7.82 (dd, 2H, quin-H), 8.20-8.22(dd, 2H, quin-H). EI-MS: $339[\mathrm{M}+1]^{+}$. Anal. Calcd for $\mathrm{C}_{16} \mathrm{H}_{8} \mathrm{ClN}_{5} \mathrm{~S}$ : C, 56.89; H, 2.39; N, 20.73\%. Found: C, 56.87, H, 2.41, N, 20.71\%.

\section{2-(4-Bromophenyl)-1-thia-3,3a,4, 9,10-pentazopentaleno[1,2-b]naphthalene (5c)}

Mp: $258-260^{0} \mathrm{C}$; IR (KBr): v $\left(\mathrm{cm}^{-1}\right) 1620(\mathrm{C}=\mathrm{N})$; ${ }^{1} \mathrm{H}$ NMR $\left(300 \mathrm{MHz}\right.$; DMSO-d $\left.d_{6}\right): \delta 7.32-$ 7.36 (dd, 2H, Ar-H), 7.70-7.72 (dd, 2H, Ar-H), 7.84-7.86 (dd, 2H, quin-H), 8.18-8.20 (dd, $2 \mathrm{H}$, quin-H). EI-MS: $383[\mathrm{M}+1]^{+}$. Anal.Calcd for $\mathrm{C}_{16} \mathrm{H}_{8} \mathrm{BrN}_{5} \mathrm{~S} ; \mathrm{C}, 50.28 ; \mathrm{H}, 2.11$; , $18.32 \%$. Found: $\mathrm{C}, 50.26 ; \mathrm{H}, 2.13 ; \mathrm{N}, 18.30 \%$.

\section{2-(p-Tolyl)-1-thia-3,3a,4, 9, 10-pentazo pentaleno[1,2-b]naphthalene (5d)}

Mp: $242-244^{0} \mathrm{C}$; I R (K Br): v $\left(\mathrm{cm}^{-1}\right) 1610(\mathrm{C}=\mathrm{N})$; ${ }^{1} \mathrm{H}$ NMR (300 MHz; DMSO- $\left.d_{6}\right): \delta 2.34$ (s, 3H, $\mathrm{CH}_{3}$ ), 7.12-7.16 (dd, 2H, Ar-H), 7.54-7.60 (dd, 2H, Ar-H), 7.72-7.78 (dd, 2H, quin$\mathrm{H}), 8.12-8.16\left(\mathrm{dd}, 2 \mathrm{H}\right.$, quin-H). EI-MS: $318[\mathrm{M}+1]^{+}$. Anal. Calcd for $\mathrm{C}_{17} \mathrm{H}_{11} \mathrm{~N}_{5} \mathrm{~S}: \mathrm{C}, 64.34$; H, 3.49; N, 22.07\%. Found: C, 64.32; H, 3.51; N, 22.06\%.

\section{Antifungal Activity}

The antifungal activity ${ }^{19}$ was assayed by sabouraud dextrose agar media plate disc diffusion method at the concentration of $50 \mu \mathrm{g}$ per disk. All the synthesized compounds were tested in vitro for their antifungal activity against microorganisms such as Candida albicans, Microsporium gypsum and microsporium canis. Each test compound was dissolved in dimethylsulphoxide (DMSO) to get a concentration of $10 \mathrm{mg} / \mathrm{mL}$. The disc $(6 \mathrm{~mm}$ in diameter was impregnated with $5 \mu \mathrm{L}$ of each test solution to get $50 \mu \mathrm{g} / \mathrm{mL}$; air dried and placed on the sabouraud dextrose agar media, previously seeded with $0.2 \mathrm{~mL}$ of broth culture of each organism for $18 \mathrm{~h}$. The plates were incubated at $22^{\circ} \mathrm{C}$ for $48 \mathrm{~h}$ and the inhibition zones were 
measured in mm. Discs impregnated with DMSO were used as a control and flucanazole disc as antifungal reference standard.

\section{Results and Discussion}

Choosing an appropriate solvent is of crucial importance for the successful organic synthesis, Room temperature ionic liquids, especially those based on 1-alkyl-3methylimidazoliumcations have shown great promise as an attractive alternative to conventional organic solvents and more attention has been currently focused on organic reactions prompted by ionic liquids ${ }^{20}$. They are nonvolatile, recyclable, non explosive, easily operable, and thermally robust ${ }^{21}$. To search for the optimal reaction solvent, the reaction of 2,3-dichloroquinoxaline $\mathbf{1}$ with substituted 2-aminothiazoles $\mathbf{2 ( a - d )}$ and substituted 2-aminothiadiazoles 3(a-d) were examined using ionic liquid such as $[\mathrm{bmim}] \mathrm{BF}_{4}$ and conventional reaction solvent such as acetic acid at different reaction temperatures. The results are summarized Table 1. It can be seen from Table 1 that the best result was obtained when the reaction was carried out in $[\mathrm{bmim}] \mathrm{BF}_{4}$ at $80^{\circ} \mathrm{C}$. It was chosen as solvent for the reactions as it is environmentally friendly and the toxic organic reagents can be avoided, Under these optimized reaction conditions a series of 3-substituted phenyl-1-thiatetrazopentaleno-[1,2-b]-naphthalene $\mathbf{4 ( a - d )}$ and 2-substituted phenyl-1-thia-pentazopentaleno-[1,2-b]-naphthalene $\mathbf{5}$ (a-d) were synthesized.

The IR spectra of compounds $\mathbf{4}(\mathbf{a}-\mathbf{d})$ and $\mathbf{5}(\mathbf{a}-\mathbf{d})$, show absorption bands within the $v$ $1620-1604 \mathrm{~cm}^{-1}$ region. They are due to symmetric vibration of $\mathrm{C}=\mathrm{N}$ group. The absence of the absorption band corresponding to amino stretching frequency of the compound substituted 2-aminothiazoles 2(a-d) and 2-aminothiadiazoles 3(a-d) clearly confirm the formation of compound 4(a-d) and 5(a-d).

\section{Antifungal Activity}

The antifungal activity was determined by the disc diffusion method at the concentration of 50 per disk. All the synthesized compounds were tested in vitro for their antifungal activity against microorganisms such as Candida albicans, Microsporium gypsum, and Microsporium canis, using flucanazole as standard antifungal. The compounds $\mathbf{4 b}$ and $\mathbf{5 b}$ were highly active. Compounds $4 \mathbf{a}$ and $\mathbf{5 a}$ were inactive, while rest of the compounds showed moderate activity.

Table 1. Results of anti-fungal activity.

\begin{tabular}{cccc}
\hline Compounds & \multicolumn{3}{c}{ Zone of Inhibition } \\
\cline { 2 - 4 } & C.albicans & M.gypsum & M.canis \\
\hline $\mathbf{4 a}$ & - & - & - \\
$\mathbf{4 b}$ & +++ & +++ & +++ \\
$\mathbf{4 c}$ & ++ & ++ & ++ \\
$\mathbf{4 d}$ & ++ & ++ & ++ \\
$\mathbf{5 a}$ & - & - & - \\
$\mathbf{5 b}$ & +++ & +++ & +++ \\
$\mathbf{5 c}$ & ++ & ++ & ++ \\
$\mathbf{5 d}$ & ++ & ++ & ++ \\
Flucanazole & +++ & +++ & +++ \\
\hline
\end{tabular}

Inactive (inhibition zone $<6 \mathrm{~mm}$ ); slightly active= ' + (inhibition zone $7-9 \mathrm{~mm}$ ); moderately active = '++' (inhibition zone 10-13 mm ); highly active = ;+++' (inhibition zone $>14 \mathrm{~mm}$ ). 
Table 1. Solvent and temperature optimization for the synthesis of $\mathbf{4 ( a - d )}$ and $\mathbf{5 ( a - d ) .}$

\begin{tabular}{cccc}
\hline \multirow{2}{*}{ Entry } & \multirow{2}{*}{$\mathrm{R}$} & \multicolumn{2}{c}{ Yield, \%/Time, $\mathrm{h}$} \\
\cline { 3 - 4 } & $\mathrm{H}$ & $92 / 1.8$ & $62 / 16$ \\
\hline $4 \mathrm{a}$ & $\mathrm{bmim}] \mathrm{VBF}_{4}$ & ${ }^{b} \mathrm{AcOH}$ \\
$4 \mathrm{~b}$ & $\mathrm{Br}$ & $93 / 1.5$ & $65 / 14$ \\
$4 \mathrm{c}$ & $\mathrm{Me}$ & $95 / 1.5$ & $67 / 14$ \\
$4 \mathrm{~d}$ & $\mathrm{H}$ & $99 / 2.2$ & $58 / 17$ \\
$5 \mathrm{a}$ & $\mathrm{Cl}$ & $93 / 2$ & $60 / 15$ \\
$5 \mathrm{~b}$ & $\mathrm{Br}$ & $94 / 2$ & $62 / 14$ \\
$5 \mathrm{c}$ & $\mathrm{Me}$ & $90 / 2.5$ & $64 / 15$ \\
$5 \mathrm{~d}$ & \multicolumn{2}{c}{$57 / 16$} \\
\hline${ }^{a}$ reaction temperature at $80^{\circ} \mathrm{C}^{b}{ }^{b}$ reaction temperature at reflux.
\end{tabular}

\section{Conclusion}

We developed an efficient and economical safe and environmentally benign procedure for synthesis of 3-substituted phenyl-1-thia-tetrazopentaleno-[1,2-b]-naphthalene 4(a-d) and 2-substituted phenyl-1-thia-pentazopentaleno-[1,2-b]-naphthalene 5(a-d) through the reaction of 2-aminothiazoles $\mathbf{2 ( a - d )}$ and 2-aminothiadiazoles 3(a-d) with 2,3-dichloro quinoxaline 1 in ionic liquid $[\mathrm{bmim}] \mathrm{BF}_{4}$ without using any catalyst. A series of the synthesized compounds $\mathbf{4 ( a - d )}$ and $\mathbf{5 ( a - d )}$ were tested for antifungal activity, the compounds $\mathbf{4 b}$ and $\mathbf{5 b}$ were found highly active against Candida albicans, microsporium gypsum and microsporium canis when compared to Flucanazole as standard antifungal agent.

\section{References}

1 Loriga M, Piras S, Sanna P and Paglietti G, Farmaco, 1997, 52, 157-166.

2 Hui X, Desrivot J, Bories C, Loiseau P M, Frank X, Hocquimiller R and Figadere B, Bioorg Med Chem Lett., 2006, 16, 815-818.

3 Sakata G, Makino K and Karasawa Y, Heterocycl., 1988, 27, 2481-2515.

4 Lindsley C W, Zhao Z, Leister W H, Robinson R G, Barnell S F, Defeozones D, Jones R E, Hartman G D, Huff J R,Huber H E and Duggan M E, Bioorg Med Chem Lett., 2005, 15, 761-764.

5 Guillon J, Philippe G, Labaied M, Sonnet P, Legar J M, Poultain P D, Sergheraet C and Christian J, J Med Chem., 2004, 47, 1997-2009.

6 Sarges R, Howerd H R, Browne R C, Label L A and Seymour P A, J Med Chem., 1990, 33, 2240-2254.

7 Dell A, William D H, Morris H R, Smith G A, Feeney J, Roberts G C K, Bailly C, Echepare S, Gago F and Waring M, J Am Chem Soc., 1975, 97, 2497-2502.

8 Sato K, Shiratori O and Katagiri K, J Antibiot., 1967, 20, 270-276.

9 Dailey S, Feast J W, Peace R J, Sage I C, Till S, Wood E L, O'Brien D, Weaver M S, Lidzey D G, Bradley D D C, Sonawane N D and Rangnaker D W, J Mater Chem., 2001, 11, 2238-2243.

10 Thomas K R J, Velusamy M, Lin J T, Chuen C H and Tao Y T, Chem Mater., 2005, 17, 1860-1866.

11 Alagarasamy V, Revathi R, Vijaykumar S and Ramseshu K V, Pharmazie., 2003, 58, 233-236.

12 Laddha S S and Bhatnagar S P, Bioorg Med Chem., 2009, 17, 6796-6802. 
13 Santagati N A, Caruso A, Cutiuli V M C and Caccamo F, II Farmaco, 1995, 50, 689-695.

14 Hori M, Lemura R, Hara H, Ozaki A, Sukamoto T and Ohtaka H, Chem Pharm Bull., 1990, 38, 681-687.

15 Abdel-Rahman A E, Bakhite E A and Al-Taifi E A, Pharmazie, 2003, 58, 372-377.

16 Ismail M M.F and Al-Azhar, J Pharm Sci., 1999, 23, 82-99. Chem Abstr., 2000, 134, 252308 AN.

17 Sakata G and Makino K, Chem Lett., 1984, 13, 323-326.

18 Makino K, Sakata G, Morimoto K and Ochiai Y, Heterocycl., 1985, 23, 2025-2034.

19 Gravestock M R and Ryley J F, Antifungal Chemotherapy Annual Reports in Medicinal Chem., 1984, 19, 127.

20 a) DZyuba S V and Bartsch R A, Angew Chem Int Ed., 2003, 42, 148.b) Wilker J S, Green Chem., 2007, 4, 73.

21 a) Welton T, Chem Rev., 1999, 99, 2071. b) Dupont J, de Sauza R F and Suarez P A Z, Chem Rev., 2002, 102, 3667. 


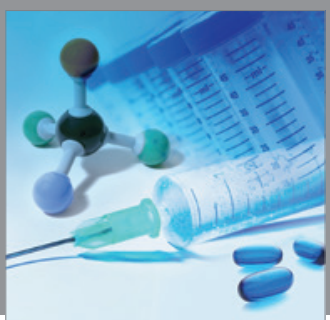

International Journal of

Medicinal Chemistry

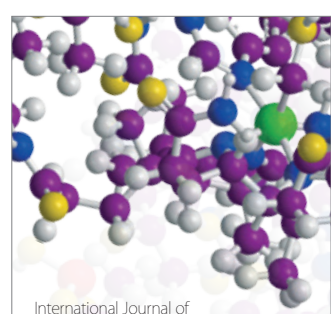

Carbohydrate Chemistry

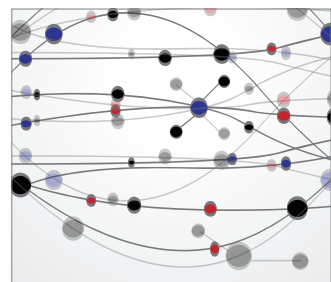

The Scientific World Journal
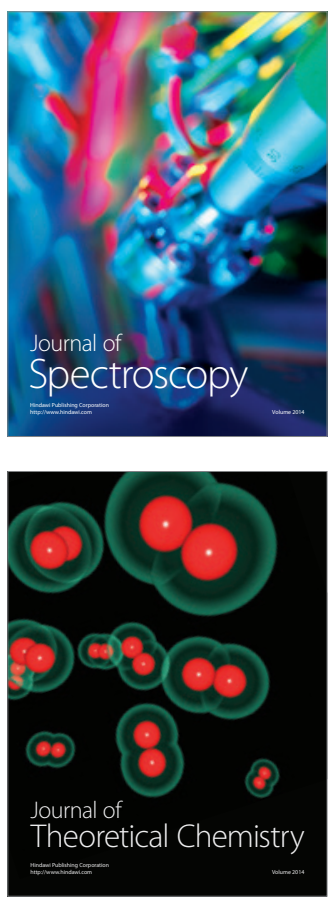
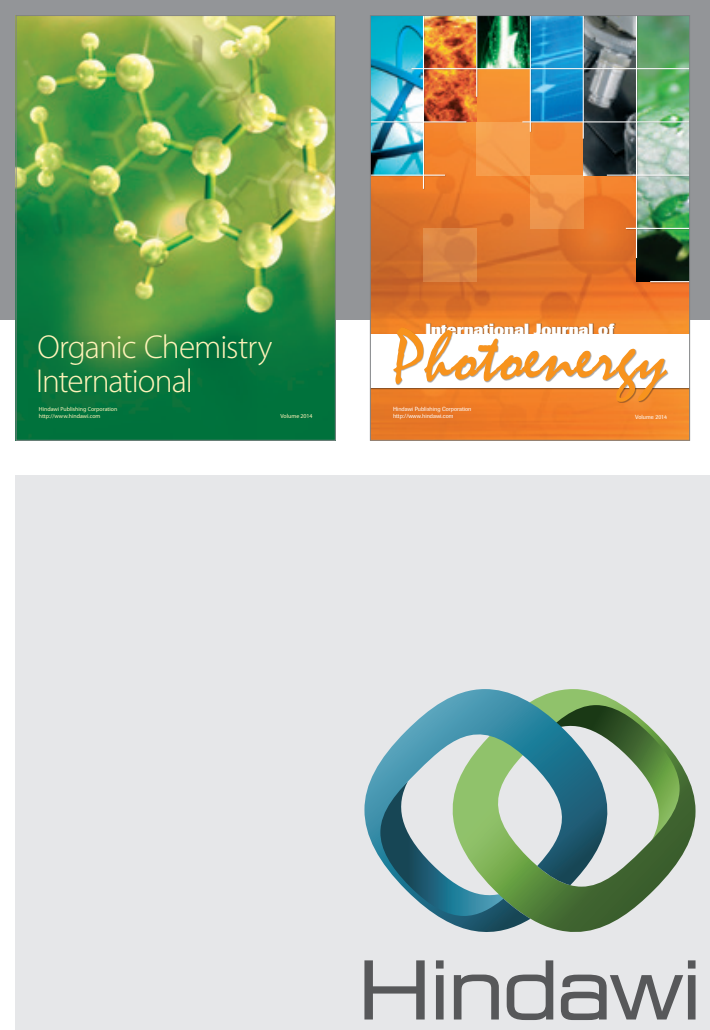

Submit your manuscripts at

http://www.hindawi.com
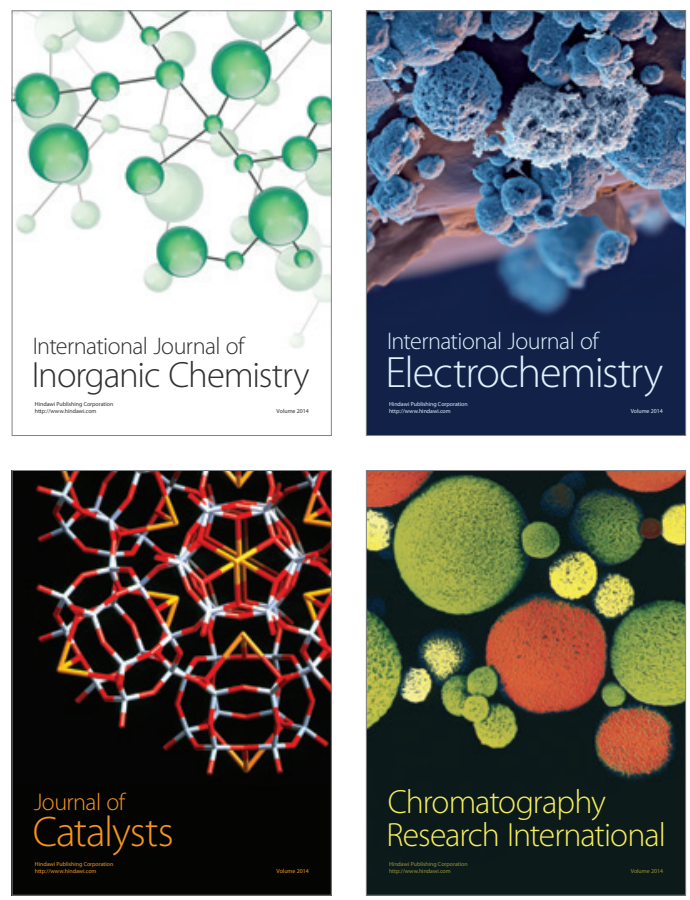
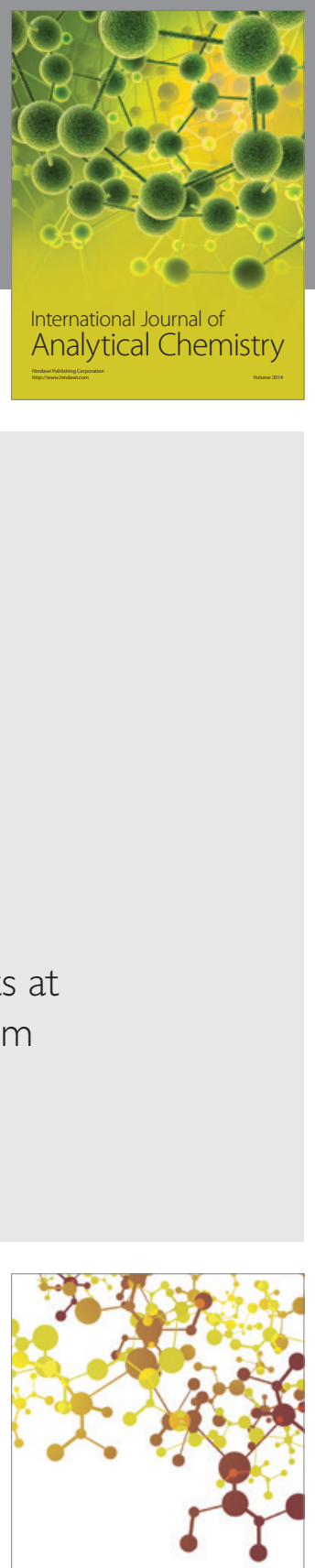

Journal of

Applied Chemistry
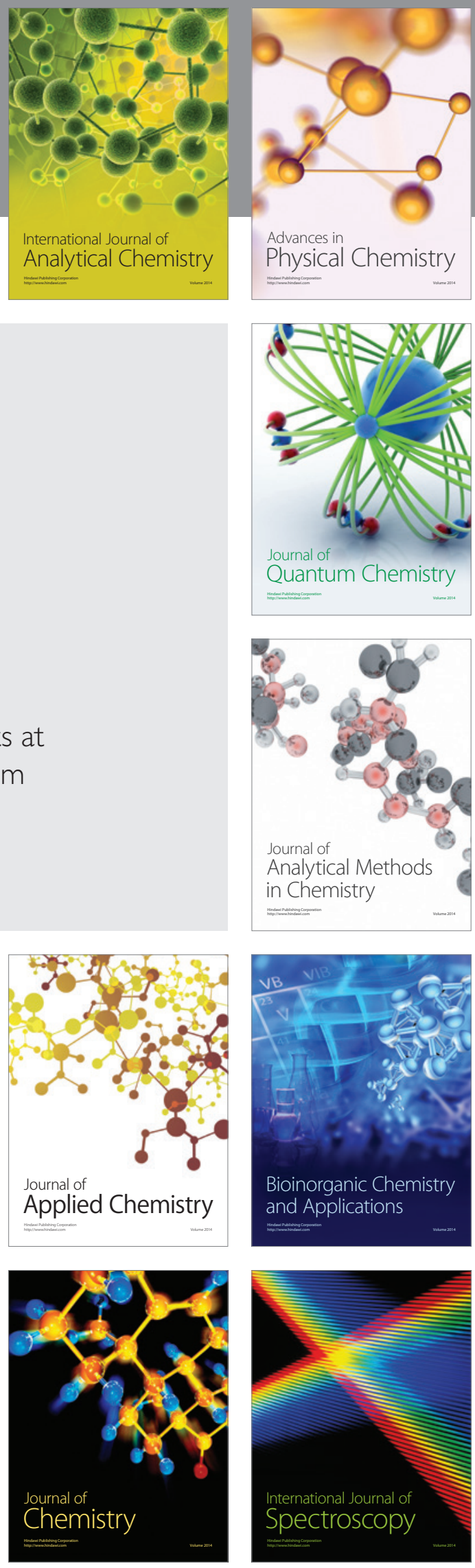